\title{
COVID-19 in older adults
}

\author{
Aman Nanda ${ }^{1}$ - Naga Venkata Rama Krishna Vura ${ }^{1} \cdot$ Stefan Gravenstein ${ }^{1,2,3}$ (])
}

Received: 23 April 2020 / Accepted: 25 April 2020 / Published online: 10 May 2020

c) Springer Nature Switzerland AG 2020

\begin{abstract}
The advent of the SARS-CoV-2 and COVID-19 pandemic has generated a lot of publications at a rapid pace. We know that older adults disproportionately suffer the most severe of COVID outcomes. Here we attempt to coalesce the key knowledge of SARS-CoV-2 biology to the disease and clinical care concepts in the context of older adults.
\end{abstract}

Keywords COVID-19 $\cdot$ SARS-CoV-2 $\cdot$ Elderly $\cdot$ Management $\cdot$ Transmission $\cdot$ Prevention

\section{Introduction}

The Corona Virus Disease-19 (COVID-19) caused by Severe Acute Respiratory Syndrome Corona Virus 2 (SARS$\mathrm{CoV}-2$ ) occurs in persons of all ages. The first individuals reported with COVID-19 were exposed in a seafood market in Wuhan, Hubei Province, China in December 2019; in the next few months, SARS-CoV-2 spread from person-toperson all across the globe, quickly evolving into a pandemic [1]. Worldwide, some 2 million people have had confirmed COVID-19 and caused more than 140,000 deaths by midApril 2020. United States now has the largest number of people of any country in whom COVID-19 has been confirmed $(>600,000)$, and deaths $(>34,000)$ [2]. Older adults with multiple co-morbidities and individuals with complex underlying health conditions suffer the severest COVID-19 outcomes [3]. In this review article, we will provide recommendations amid this pandemic to protect the vulnerable older adult population.

Stefan Gravenstein

Stefan_Gravenstein@brown.edu

1 Division of Geriatric and Palliative Medicine, Department of Medicine, Rhode Island Hospital, Warren Alpert Medical School of Brown University, 593, Eddy Street, POB 438, Providence, USA

2 Department of Health Services Policy and Practice, School of Public Health, Brown University, Providence, USA

3 Providence Veterans Administration Medical Center, Providence, USA

\section{Pathogenesis}

SARS-CoV-2 is a positive-sense single-stranded RNA coronavirus in the family of Coronaviridae, along with SARSCoV, PC4-227, and BtKY72 [4]. Two different strains L and $\mathrm{S}$ are detected in circulation; the pathogenesis and clinical implications of these strains are not well characterized. For initial infection and cell entry, SARS-CoV-2 binds to angiotensin-converting enzyme 2 (ACE 2) receptor like SARS-CoV. The cellular entry of SARS-CoV-2 depends upon ACE2 and TMPRSS2 and can be blocked by a protease inhibitor [5]. The ACE 2 surface receptors are present in multiple tissues: basal layer of non keratinizing epithelium in oropharynx, endothelial cells on arterial walls, smooth muscle, heart, alveolar cells, enterocytes in the brush border of the entire length of the small intestines, and proximal tubules of the kidneys [6]. However, only the binding sites in the small intestines and lungs are readily available for the initial binding of SARS-CoV-2 at the time of inoculation, making these the likely portals of initial entry. The epithelial cells of oral and nasal mucosa lack ACE 2 expression, and this may help explain the limited upper respiratory symptoms in COVID-19 [6]. The small intestine and pulmonary binding sites correspond to clinical symptoms of diarrhea and later nausea and cough early in the course, before the likely viremic spread to lesser accessible sites such as the nasopharyngeal area to cause the later symptoms pharyngitis and eventually anosmia, myocarditis, vasculitis, and ARDS. In another study, high blood levels of cytokines and chemokines were noted in patients with SARS-CoV-2 infection that included IL1- $\beta$, IL1RA, IL7,IL8, IL9, IL10, basic FGF2, GCSF, GMCSF, IFN $\gamma$,IP10, MCP1, MIP1 $\alpha$, MIP1 $\beta$, 
PDGFB, TNF $\alpha$, and VEGFA [7]. These molecules also relate to the elevation of acute phase reactants and cytokine storm in those who develop a hyper-immune response as they progress to more severe disease usually a week or so after initial presentation.

\section{Transmission}

The unique features of transmission of SARS-CoV-2 are one of the leading contributors of COVID-19 pandemic. It spreads by direct contact with large respiratory droplets from person to person and indirect contact with infectious droplets or secretions that have deposited on surfaces in the environment. Inoculation often occurs with the transfer touching of infected material on one of these surfaces and then touching a mucosal surface, such as the mouth, nose or eyes. The virus does not typically produce sneezing that can produce an aerosol in which the virus might remain viable for hours and be transmitted by inhalation. The virus is most likely and more often to be transferred from surfaces where it can remain viable for up to days. It has the longest lifespan on high gloss surfaces, less on hard surfaces (days), paper, synthetics and very short on cotton and copper [8-10]. Another important aspect of its alarming spread is a long incubation period (range 2-27 days, median 5-6 days), where 97.5\% of those who develop symptoms will do so within 11.5 days. It has been estimated that $50 \%$ or more of asymptomatic persons will shed virus [11].

The virus' predilection for the lower respiratory tract is especially problematic for frail older adults who receive direct contact and care by asymptomatic healthcare workers, a feature that is a set-up for a period of clinically silent spread within long-term care facilities before clinical presentation of COVID-19 [3].

\section{Clinical manifestations}

The most common symptoms reported by patients with COVID-19 include fever, fatigue, and dry cough [12]. Less common symptoms include headache, anosmia, cough with sputum production, joint pains, chills, nausea, vomiting, and diarrhea. However, older adults often present atypically, such as with sore throat, delirium and unexplained hypoxia, tachycardia or tachypnea. The illness caused by SARSCoV-2 can range from mild to critically ill. Mild symptoms can be reported in some $80 \%$ of the patients, but are likely much more common in younger populations who may be undercounted in these estimates because they do not seek or are not offered testing. Severe symptoms with shortness of breath and lung involvement are reported in $14 \%$ of the patients, and 5\% develop critical illness. The case fatality rate varies from 2.3 to $14.8 \%$ depending on the demographics of the nation or region, age, severity of the disease and comorbidities. Older adults of the 70-80-year-old age group have a case fatality rate of $8.0 \%$, and older adults above age 80 have a case fatality rate of $14.8 \%$ [13]. With age come additonal pre-existing conditions, which makes older adults more susceptible to developing a severe infection. Male sex, and comorbidities such as cardiovascular disease, diabetes mellitus, hypertension, chronic kidney disease, obesity, and chronic lung disease are associated with the development of severe disease $[3,7,14]$. Critically ill patients develop significant complications such as acute respiratory distress syndrome (ARDS) requiring prolonged ventilatory support, cardiac injury, cardiac rhythm disorders, shock and death.

Laboratory findings include leukopenia, lymphopenia, elevated liver enzymes, ferritin, lactate dehydrogenase, prothrombin time, and d-dimer [12]. Patients with elevated d-dimer and severe lymphopenia have greater mortality [15]. Imaging findings on chest radiographs include patchy bilateral opacities or consolidations. Chest computed tomography (CT) findings include bilateral ground-glass opacities involving the periphery and base of the lungs [12].

\section{Diagnosis}

COVID-19 diagnosis should be considered in any older adult who develops a new-onset fever or respiratory symptoms such as dry cough or breathing difficulty in the setting of community spread. However the older frail patients may have atypical presentations like unexplained delirium, hypoxia, tachycardia, and tachyapneoa. Definitive diagnosis currently relies on reverse-transcription polymerase chain reaction (RT-PCR) of SARS-CoV-2 on a nasopharyngeal swab, although other tests are rapidly becoming available [16]. Testing for seasonal influenza and other respiratory pathogens may or may not be done simultaneously based on prevalence of their co-circulation in the community. A study that analyzed the results of specimens obtained from various methods showed increased positive test results from samples obtained by bronchoalveolar lavage (BAL) fluid at 93\% compared to $63 \%$ and $72 \%$ positive test results from samples obtained from sputum and nasal swabs, respectively [17].

\section{Treatment}

Currently, evidence for effective treatment for Covid-19 is rapidly evolving. Presently, treatment is supportive. All cases or suspected cases should be isolated until effective treatments or vaccines become available. Mild cases can be managed at home; while, those who develop hypoxia, respiratory distress or cytokine storm will require higher levels of 
care and potentially ventilator support. Therefore, the most important strategy remains as primary prevention of initial infection and halting spread from those who are infected.

\section{Guidelines for prevention}

Guidelines to prevent the spread of the disease can be divided into three categories: Individual, Long-term care facility and Ambulatory care office [16].

\section{Individual level}

Recommendations given to the general public to counteract the rapid spread of the virus are frequent hand washing, avoid touching eyes, nose, and mouth, social distancing (atleast $6 \mathrm{ft}$, preferably $>13 \mathrm{ft}$ ), staying at home, and wearing a cloth face covering when going outside. Widespread use of face masks and social distancing have a vital role in decreasing the virus' pace of spread.

\section{Long-term care facility}

These recommendations can be divided into three main groups: Organization/Administrators, Health care workers, and residents of the facility.

\section{Organization level}

The most important is to train all health care workers on the use of personal protective equipment (PPE), especially donning and doffing procedures. The administrators should stock and track supplies of PPE, including surgical and N95 masks, isolation gowns, face shields, goggles, and non-sterile gloves. They should plan for PPE shortages and reach out to nearby facilities and their local department of health to harmonize approaches that help to maintain PPE supplies while protecting their workforce and facility residents. While the use of telemedicine services by clinicians for residents should be encouraged to reduce vectoring of disease between provider, staff and residents, its implementation adds burden to frontline staff that needs to be addressed. The visitation of all nonessential visitors including family members should be stopped. Every person including all staff-from cooks and housekeeping to nurses and adminstrators - entering the facility should be screened for elevated temperature or presence of symptoms. In addition, staff should also be asked if they have had any contact with a COVID-19-positive (or suspected) person. Maintaining a list of staff who work in any other facility is important in case there is an outbreak in that facility; such staff are often per diem or among the lowest paid workers. Moonlighting in another facility should be discouraged. Thus, as much as possible, avoiding the employment of temporary or per diem staff because of their risk as a nosocomial vector from another facility is high [3]. Try to limit the staff to work on the same floor or same residents every day. All medicines should be reviewed and unnecessary medicines like vitamins should be discontinued to reduce the number of contacts between nursing staff and residents. Use of nebulizers and devices that can aerosolize virus (e.g., CPAP) should be strongly discouraged or avoided altogether. Active monitoring for potentially infected patients and strict implementation of appropriate infection prevention and control measures are needed to prevent COVID-19 in long-term care facilities.

\section{Health care workers}

Guidance for health care workers includes avoiding coming to the facility with respiratory symptoms or with elevated temperature. Following strict hand hygiene with hand wash for every person entering the facility (alcohol based or $20 \mathrm{~s}$ with soap and water). Do not shake hands, touch, or hug residents or other staff members. Follow current guidelines for protective equipment recommended for close contact with residents.

\section{Residents}

Guidelines at resident level include avoiding group dining, group therapies, and recreational activities involving large groups. Halt all visitors, including close family members. Postpone non-urgent outpatient visits. Use of masks is recommended for all residents who leave their rooms, and for those with confirmed or suspected of SARS-CoV-2 infection, whether symptomatic or not, should be isolated with contact precautions. Staff entering rooms will benefit from the use of available personal protective equipment and education on the donning and doffing of this equipment.

\section{Ambulatory care office}

Ambulatory care office includes educating the patients and staff of all the guidelines for Individuals as noted above. Promote to see patients by tele-visit except urgent visits. Avoid inviting patients to come to the office if there is any suspicion of COVID-19 infection unless the office is absolutely prepared to handle those patients safely. Mask patients who come in the office and mask staff while there is interaction between staff, and between staff and patients.

\section{Conclusions}

The current pandemic caused by SARS-CoV-2 has led the world to come to a stop. The world has also witnessed the importance of health care workers being on the front lines 
defending the virus from spreading as well as risking their own lives to treat the sick and vulnerable population. Use of updated guidance from medical societies regarding strategies to decrease the spread of infection until we find effective drugs and vaccines will translate into decreased mortality of person of all ages, especially the vulnerable older adults living in the community and in the long-term care facilities. Effective prevention strategies implemented by infection control teams in the long-term care facilities will ease the strain on local acute care hospitals.

Funding The authors do not have any commercial or financial conflicts of interest or any funding sources.

\section{Compliance with ethical standards}

Conflict of interest The authors declare that they have no conflict of interest.

Statement of human and animal rights This article does not contain any experimental study with humans or animals performed by the authors.

Informed consent Informed consent is not applicable.

\section{References}

1. Li Q, Guan X, Wu P et al (2020) Early transmission dynamics in Wuhan, China, of novel coronavirus-infected pneumonia. N Engl J Med 382:1199-1207. https://doi.org/10.1056/NEJMoa2001316

2. COVID-19 Map (2020) Johns hopkins coronavirus resource center. https://coronavirus.jhu.edu/map.html. Accessed $14 \mathrm{Apr}$ 2020

3. McMichael TM, Currie DW, Clark S et al (2020) Epidemiology of Covid-19 in a long-term care facility in King County, Washington. N Engl J Med. https://doi.org/10.1056/NEJMoa2005412 (e-pub)

4. Gorbalenya AE, Baker SC, Baric RS et al (2020) Severe acute respiratory syndrome-related coronavirus: the species and its viruses - a statement of the Coronavirus Study group. BioRxiv. https://doi.org/10.1101/2020.02.07.937862

5. Hoffmann M, Kleine-Weber H, Schroeder S et al (2020) SARSCoV-2 cell entry depends on ACE2 and TMPRSS 2 and is blocked by a clinically proven protease inhibitor. Cell 181:271-280. https ://doi.org/10.1016/j.cell.2020.02.052

6. Hamming I, Timens W, Bulthuis MLC et al (2004) Tissue distribution of ACE2 protein, the functional receptor for SARS coronavirus. A first step in understanding SARS pathogenesis. J Pathol 203:631-637. https://doi.org/10.1002/path.1570.

7. Huang C, Wang Y, Li X et al (2020) Clinical features of patients infected with 2019 novel coronavirus in Wuhan, China. Lancet 395:497-506. https://doi.org/10.1016/S0140-6736(20)30183-5

8. Van Doremalen N, Bushmaker T, Morris DH et al (2020) Aerosol and surface stability of SARS-CoV-2 as compared with SARSCoV-1. N Engl J Med 382:1564-1567. https://doi.org/10.1056/ NEJMc2004973

9. Ong SWX, Tan YK, Chia PY, Lee TH, Ng OT, Wong MSY, Marimuthu K (2020) Air, surface environmental, and personal protective equipment contamination by severe acute respiratory syndrome Coronavirus 2 (SARS-CoV2) from a symptomatic patient. JAMA. https://doi.org/10.1001/jama.2020.3227 (e-pub)

10. Santarpia JL, Rivera DN, Herrera V et al (2020) Transmission potential of SARS-CoV-2 in viral shedding observed at the University of Nebraska Medical Center. MedRxiv. https://doi. org/10.1101/2020.03.23.20039446 (Preprint posted March 26, 2020)

11. Lauer SA, Grantz KH, Bi Q et al (2020) The incubation period of Coronavirus disease 2019 (COVID-19) from publicly reported confirmed cases: estimation and application. Ann Intern Med. https://doi.org/10.7326/M20-0504 (e-pub)

12. Wang D, Hu B, Hu C et al (2020) Clinical characteristics of 138 hospitalized patients with 2019 novel Coronavirus-infected pneumonia in Wuhan, China. JAMA 323:1061-1069. https://doi. org/10.1001/jama.2020.1585

13. Wu Z, McGoogan JM (2020) Characteristics of and important lessons from the Coronavirus disease 2019 (COVID-19) outbreak in china: summary of a report of 72,314 cases from the chinese center for disease control and prevention. JAMA 323:1239-1242. https://doi.org/10.1001/jama.2020.2648

14. Lighter J, Phillips M, Hochman S et al (2020) Obesity in patients younger than 60 years is a risk factor for Covid-19 hospital admission. Clin Infect Dis. https://doi.org/10.1093/cid/ciaa415

15. Chen N, Zhou M, Dong X et al (2020) Epidemiological and clinical characteristics of 99 cases of 2019 novel coronavirus pneumonia in Wuhan, China: a descriptive study. Lancet 395:507-513. https://doi.org/10.1016/S0140-6736(20)30211-7

16. CDC-Centers for Disease Control and Prevention (2020) Coronavirus disease 2019 (COVID-19). https://www.cdc.gov/ coronavirus/2019-ncov/lab/guidelines-clinical-specimens.html. Accessed 12 Apr 2020 (Published February 11, 2020)

17. Wang W, Xu Y, Gao R et al (2020) Detection of SARS-CoV-2 in different types of clinical specimens. JAMA. https://doi. org/10.1001/jama.2020.3786

Publisher's Note Springer Nature remains neutral with regard to jurisdictional claims in published maps and institutional affiliations. 